\title{
Changes in Maternal Lifestyle during Ramadan Altered Placental Development
}

\author{
Nibras S. Baanter' ${ }^{1}$ Amel A. A. Elsayed ${ }^{2}$, Khaldoon Aljerian³, \\ Waleed M. Aldahmash ${ }^{1}$, Wedad S. Al-Qahtani ${ }^{1}$, Saleh H. Alwasel ${ }^{*}$ \\ ${ }^{1}$ Zoology Department, College of Science, King Saud University, Riyadh, Saudi Arabia \\ ${ }^{2}$ Obstetrics and Gynecology Department, College of Medicine, King Saud University, Riyadh, Saudi Arabia \\ ${ }^{3}$ Department of Pathology, College of Medicine, King Saud University, Riyadh, Saudi Arabia \\ Email: *salwasel@ksu.edu.sa
}

How to cite this paper: Baanter, N.S., Elsayed, A.A.A., Aljerian, K., Aldahmash, W.M., Al-Qahtani, W.S. and Alwasel, S.H. (2020) Changes in Maternal Lifestyle during Ramadan Altered Placental Development. Advances in Reproductive Sciences, 8, 126-142.

https://doi.org/10.4236/arsci.2020.82011

Received: April 11, 2020

Accepted: April 25, 2020

Published: April 28, 2020

Copyright $\odot 2020$ by author(s) and Scientific Research Publishing Inc. This work is licensed under the Creative Commons Attribution International License (CC BY 4.0).

http://creativecommons.org/licenses/by/4.0/

\begin{abstract}
People born with low birth weight are at a greater risk of developing later life diseases such as hypertension, diabetes and cancer. Recent studies have pinpointed the placenta as a critical factor involved in developmental programming. Changes in maternal lifestyle or dietary habits can alter placental development and increase the risk of developmental programming of adult diseases. Saudi people, including pregnant women, change their lifestyle and eating habits during the holy month of Ramadan. Previous studies found that the exposure to Ramadan lifestyle reduces placental weight; however, effects on other placental aspects remained unknown. We aimed to further examine the effects of exposure to Ramadan lifestyle on full-term placental morphometrics, histology and gene expression of key glucose transporters. To examine this, fresh placentas were collected from 60 healthy Saudi women. Samples were equally classified into two groups; not exposed to Ramadan lifestyle (control) or exposed to Ramadan lifestyle in the first. Placental weight, length and breadth were recorded and placental surface area was calculated. Placental tissue was processed and stained with eosin and hematoxylin for histological examination. Apoptosis was assessed using TUNEL assay. The gene expression of the glucose transporters GLUT1 and GLUT3 was evaluated. The results show that women exposed to Ramadan lifestyle have more elongated placentas with less central cord insertion. Placental weight and surface area were significantly lowered in women exposed to Ramadan lifestyle. Placental length was not affected but the breadth was significantly smaller in than control. Placentas exposed to Ramadan lifestyle had fewer and less-developed syncytial knots and thicker syncytiotrophoblast cells. Apoptosis was detected in placentas exposed to Ramadan lifestyle. GLUT1 mRNA expression was unaltered, but GLUT3 was increased compared to control group. These findings suggest that changes
\end{abstract}


in maternal lifestyle during Ramadan can alter placental structure at morphometric, histological and molecular levels. These structural changes are indication of placental adaptations for a suboptimal maternal environment. Such adaptations have been linked to adult diseases in various populations worldwide. Further studies are required to evaluate the possible link between exposure to Ramadan lifestyle and the risk of developing adulthood chronic diseases in the Saudi population.

\section{Keywords}

Placenta, Ramadan, Glucose Transporter, Fetal Programming

\section{Introduction}

The placenta is a temporary organ that connects the fetus to the mother in mammals. One of the main functions of the placenta is to provide the growing fetus with adequate levels of nutrients [1]. Human placenta starts to develop immediately after implantation and continues to develop until birth. Placenta development is influenced by maternal environment. For example, the weight of a full-term placenta can range from 400 to 800 grams depending on maternal dietary conditions. Similarly, the fetoplacental ratio (percentage of placental weight/fetal weight) can also have a very wide range. Typically, the placenta is described as a round- or oval-shaped organ; however, other shapes such as triangular, rectangular, irregular or multilobular are not infrequent [1] [2]. Thus, the human placenta is known for its high plasticity and ability to adapt to maternal metabolic challenges [3].

Villi are the functional units in the placenta. The chorionic villous tree is covered by a trophoblast layer that controls material exchanges between the mother and her fetus. The trophoblast layer consists of two distinct layers; the first is the cytotrophoblast layer which stands on the basement membrane. Cells of this layer are cuboidal to oval-shaped with relatively large and round nuclei. These cells are undifferentiated and the majority of them disappear at the third trimester [4]. The second is the syncytiotrophoblast cell which covers all villi and is in a direct contact with maternal blood. This surface area of this layer in a full-term placenta is about $12 \mathrm{~m}^{2}$ in total [5]. Cellular membranes between syncytiotrophoblast cells disappear at some stages which leads to syncytial nuclei aggregation and the formation of syncytial knots. The number of syncytial knots continues to increase as gestation proceeds. It is routinely examined and used as a marker of placental maturity and well-being [1]. Some of the syncytial knots can develop to form apoptotic bodies [6]. Cellular apoptotic can be assessed by TUNEL assay, a method that can detect fragmented DNA.

Fetal growth depends on the availability of nutrients in maternal blood and the ability of the placenta to transfer nutrients according to fetal demands [7]. People born with low birth weight are at a greater risk of developing later life diseases such as hypertension [8], heart diseases [9], diabetes [10], chronic kidney disease 
[11] and different types of cancer [12] [13]. This phenomenon is known as fetal programming of diseases or developmental origin of health and disease [14]. Recent studies focused on the placenta as a critical factor in fetal programming. The strong relationship between placental weight and birth weight has been well documented in early studies [15] [16]. Reports show that placental weight and the fetoplacental ratio could be altered by various maternal factors including dietary intake, anemia, high blood pressure or smoking [17]. Low placental weight can lower birth weight, leading to long-term health consequences [18] [19]. Not only is it the placental weight that matters but also the placental shape and surface area, which reflects the exchange surface between maternal and fetal blood. Maternal undernutrition has been reported to reduce placental surface area [20] and has also been flagged as an early marker of fetal programming. Reduced placental surface area per se can lead to hypertension in later life [20]. Assuming an elliptical shape, the surface area is calculated as length $\times$ breadth $\times \pi / 4$. Since the placental disk tends to be oval-shaped rather than round, some studies focused not only on the crude surface area but also on the roles of the length and the breadth. Barker et al. [13] found that an oval placental surface at birth is associated with later colorectal cancer. Another study found that the breadth of the placental surface but not the length was associated with body size at birth [21]. It was speculated that the tissue along the breadth may play a more important role than the tissue along the length in transporting nutrients from the maternal to the fetal side [13].

We studied over 7000 birth records in the main hospital of Unizah City, central Saudi Arabia, and found that the average birth weight is similar to European values but that the average placental weight and fetoplacental ratio were lower. This suggests that the efficiency of placenta in Saudi women is increased [22]. However, the mechanisms of material transportation across the placental barrier are still not clear and need further investigation. Glucose is the principal energy substrate for the fetus, and thus, it is essential for normal development and growth. Fetal consumption of glucose rapidly increases toward term due to the almost 20 -fold increase in fetal weight during the second half of gestation [23]. Different isoforms of glucose transporters (GLUTs) are present in the placenta. GLUT1 and GLUT3 have been reported to be responsible for the majority of fetal glucose uptake [24]. GLUT1 is found in the apical microvillous membrane of syncytiotrophoblast cells, whereas GLUT3 is present in the arterial vascular endothelium (Illsley NP 2000). It is thought that the mRNA expression of GLUT1 and GLUT3 is influenced by the maternal current diet [25].

Changes in maternal diet consumption were reported to affect both the placental ability to deliver elements and fetal growth [26]. Ramadan is the fasting and holy month in Islam. Adult Muslims abstain from eating, drinks or smoking from dawn to sunset. It is the ninth month of the year and can fall in different seasons because the year in the Islamic calendar, which depends on the moon, is 10 days shorter than the Gregorian year. Accordingly, the daytime of Ramadan 
in Saudi Arabia ranges from 10 to 15 hours per day depending on the season. The effects of intermittent fasting on pregnancy are controversial. While some studies suggest that fasting has positive impacts on the heart [27] and brain [28], other studies reported that fasting affects nutrient storage and reduce blood glucose in normal adults [29]. Sakar et al. [30] examined pregnant Turkish women who were exposed to Ramadan fasting during their second or third trimester. Using Doppler ultrasound, they found significant retardation in fetal development parameters including biparietal diameter, head circumference, femur length and estimated fetal weight. Fasting is not the only change occurs in Ramadan. In fact, lifestyle is markedly changed during Ramadan month in Saudi Arabia. People change their daily activities, eating habits and meals, sleeping and working hours.

Although pregnant Muslim women are exempt from fasting, the majority of them prefer to fast all or at least some days of Ramadan. Glazier et al. [31] estimated that the 230 million Muslim women worldwide with a fertility rate of $3.1 \%$ have the potential for up to 535 million babies in each generation to be exposed in utero to the Ramadan lifestyle. In retrospective studies, it was found that the changes in lifestyle during Ramadan reduce the placental weight but did not affect the baby weight [22] [32]. In the current prospective study, we hypothesized that Ramadan lifestyle may alter blood glucose level during daytime and night in pregnant Saudi women leading to placental compensations in terms of its morphometrics, histology or glucose transporter expression.

\section{Material and Methods}

The study was approved by the Ethical committee (Ref. No. 16/0215/IRB; 2016), King Saud Medical City, King Saud University, Riyadh, Saudi Arabia. A well-written informed consent letter was obtained from all participants in the study.

\subsection{Placental Collection}

A total of 60 fresh placentas were collected from Saudi pregnant women who attended the Obstetrics and Gynecology Department, King Saud Medical City, between July 2016 and July 2017. Complicated pregnancy, multiple pregnancy, preor post-term and cesarean delivery were excluded from the study. Pregnant women were classified into two groups according to Ramadan exposure: not exposed to Ramadan (Control, $n=30$ ) or exposed to Ramadan lifestyle (ERL, $n=30$ ).

\subsection{Placental Morphometric Measurements}

Placentas were immediately flush-washed with buffered saline to remove any blood clots. The placenta was photographed for shape and insertion point assessment. A paper measuring tape was used to measure the length and breadth of the placenta. The maximal diameter of the surface was considered as the length. The short diameter that bisected to the length at right angle was considered as the breadth. Placental weight was measured to the nearest gram using a digital scale. 


\subsection{Tissue Processing for Histological Examination}

Four full-depth placental tissue samples were collected from each quarter of the placental disk. All samples were fixed in $10 \%$ neutral buffered formalin and processed to generate paraffin blocks following standard protocols. Thin sections $(5 \mu \mathrm{m})$ were deparaffinized, rehydrated and stained with hematoxylin and eosin.

\subsection{TUNEL Assay}

Tissue sections were permeabilized using $0.1 \%$ Triton X-100 with sodium citrate. This was followed by addition of $0.3 \%$ pepsin in $\mathrm{HCl}(\mathrm{pH}=2)$ for 5 minutes at $37^{\circ} \mathrm{C}$. Sections were submerged in citrate buffer and placed in microwave at 750 Watt for 45 second followed by two washing steps with phosphate buffered saline. Following the manufacturer's instructions, in Situ Cell Death Detection kit (Roche Diagnostics, Germany) was used for TUNEL staining. Positive control sample was treated recombinant DNase-I for $10 \mathrm{~min}$ at room temperature in order to induce DNA fragmentation. Some slides treated without TdT as negative controls. All sections were stained with Hoechst, washed in TE buffer and mounted in 50\% glycerol/TE. Sections were photographed using Nikon TE 2000 fluorescence microscope connected to Nikon DS-cooled camera.

PBS, stained with Hoechst, washed in TE buffer for $10 \mathrm{~min}$ and mounted in $50 \%$ glycerol/TE. The anterior parts of adult specimens containing the ovaries were fixed in neutral buffered formalin for $18-24 \mathrm{~h}$ and were preserved in $70 \%$ alcohol. Paraffin sections were prepared at intervals of $3 \mu \mathrm{m}$ and mounted on slides that were dried overnight at room temperature to remove any water that might be trapped under the sections. Before proceeding with the staining protocol, the slides were deparaffinized, rehydrated and washed in PBS. Paraffin sections were permeabilized in $0.1 \%$ Triton $\mathrm{X}-100$, with $0.1 \%$ sodium citrate, then treated with pepsin $(0.3 \%$ in $\mathrm{HCl}, \mathrm{pH} 2)$ for $5 \mathrm{~min}$ at $37^{\circ} \mathrm{C}$. Slides were placed in plastic jars containing 0.1 citrate buffer, $\mathrm{pH} 6$, for microwave irradiation at 750 $\mathrm{W}$ for $45 \mathrm{~s}$, and were then rinsed twice with $1 \times$ PBS. TUNEL staining was performed with an in Situ Cell Death Detection Kit, TMR red 12156792910 (Roche Diagnostics, Mannheim, Germany) following the manufacturer's instructions. The positive control was incubated in the same conditions as the rest of the samples but after pretreatment with recombinant DNase I (Roche Diagnostics) for $10 \mathrm{~min}$ at room temperature before the reaction mixture. Negative controls were prepared by the omission of TdT in the reaction mixture. Samples were then rinsed.

\subsection{Gene Expression}

Fresh placental biopsies $(50 \mathrm{mg}$ ) were obtained from each placental quarter to extract total RNA using $5 \mathrm{ml}$ of TRIzol reagent (Invitrogen Life Technologies, Carlsbad, CA, USA). The extract was resuspended in $200 \mu$ l of RNAse-free water. RNA concentrations were quantified by optical density measurements at 260 and $280 \mathrm{~nm}$. The purity and integrity of RNA were checked by $2 \%$ agarose gel 
electrophoresis. cDNA was synthesized from $2 \mu \mathrm{g} / \mu \mathrm{l}$ total RNA using the QuantiTect Reverse Transcription kit (Qiagen, Limburg, Netherlands). After genomic DNA elimination, the RNA sample was used for reverse transcription using a master mix (Quantiscript Reverse Transcriptase), Quantiscript RT Buffer, and RT Primer Mix (Qiagen). An aliquot of each tube was added to the real-time polymerase chain reaction (PCR) mix. A two-step process was employed for cytokine mRNA quantification (SYBR Green PCR Master Mix kit, Qiagen). Following the synthesis reaction, 2X QuantiTect SYBR Green PCR Master Mix was thawed with the synthesized cDNA. The procedure was repeated using specific forward and reverse primers for humans (synthesized by Invitrogen, San Diego, CA), where GAPDH was used as a PCR internal control gene for the target genes as described by Janzen and co-authors [33] (Table 1). The amplifications were performed using a real-time PCR instrument (7500, Applied Biosystems, Grand Island, USA) with an initial activation at $95^{\circ} \mathrm{C}$, denaturation at $94^{\circ} \mathrm{C}$, annealing at $60^{\circ} \mathrm{C}$, and extension at $72^{\circ} \mathrm{C}$ for 35 cycles. The amplification was repeated three times for each sample. Gene expression was normalized to $\beta$-actin in each sample.

\subsection{Statistical Analysis}

Data were statistically analyzed using SPSS software. T-test was performed to examine the differences between groups. Differences were considered statistically significant when $p<0.05$. All data are presented as the mean \pm standard deviation.

\section{Results}

\subsection{Morphological and Morphometric Changes}

Overall, the shape of the placenta tended to be more elongated in women who were exposed to Ramadan lifestyle compared to control group. The insertion point of the umbilical cord tended to be less central in placentas from women who were exposed to Ramadan lifestyle in comparison to control placentas.

Placental weight in women exposed to Ramadan lifestyle was significantly lower than control (Figure 1). Placental length was comparable between the two groups (Figure 2); however, placental breadth was significantly reduced in women exposed to Ramadan lifestyle during gestation (Figure 3). The placental surface area was significantly smaller in women exposed to Ramadan lifestyle in comparison to control group (Figure 4).

Table 1. Prob and primer sequences (5' to $3^{\prime}$ ) used for PCR. Annealing temperature (An. Tm.) was shown for each gene.

\begin{tabular}{cccc}
\hline $\begin{array}{c}\text { Gene } \\
\text { name }\end{array}$ & Forward & Reverse & $\begin{array}{c}\text { An. } \mathbf{T m} . \\
\left({ }^{\circ} \mathrm{C}\right)\end{array}$ \\
\hline$\beta$-Actin & CTGGCACCCAGCACAATG & GCCGATCCACACGGAGTACT & 60 \\
GLUT1 & ATGGCGGGTTGTGCCATA & ATAGGACATCCAGGGTAGCTGCTCC & 60 \\
GLUT3 & CAGGCACACGGTGCAGATAG & GCAGGCTCGATGCTGTTCAT & 60 \\
\hline
\end{tabular}




\subsection{Histological Changes in the Placenta}

Figure 5(A) shows the normal histology of placentas that were not exposed to Ramadan lifestyle. The syncytiotrophoblast layer is well-developed and cell-cell fusion can be easily detected. Because of cellular fusion, nuclei are pushed to the corner leaving a very thin syncytial cytoplasmic layer. The syncytiotrophoblast nuclei

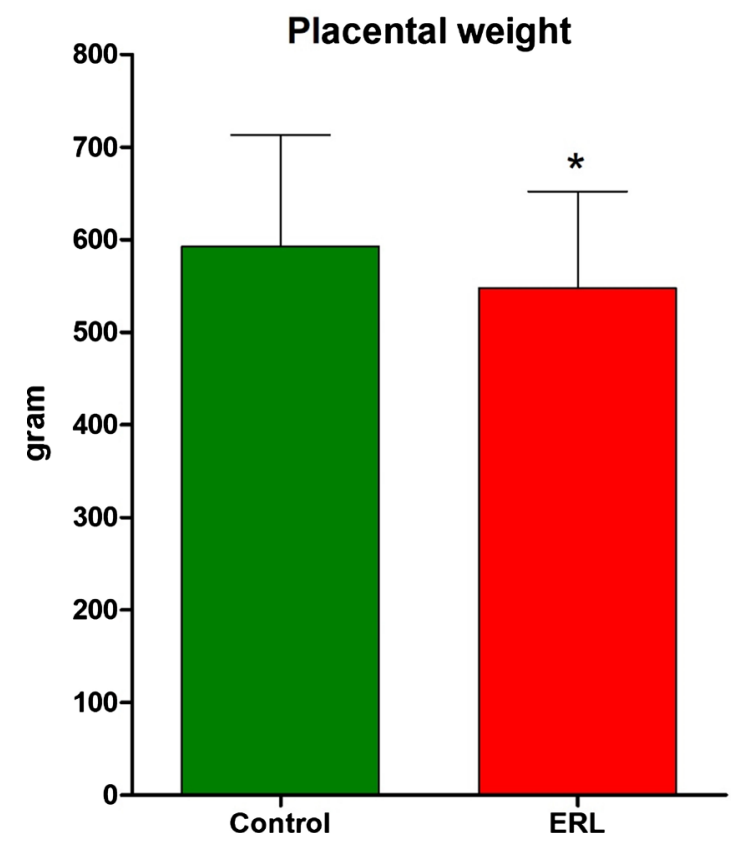

Figure 1. Placental weight was significantly reduced in women who exposed to Ramadan lifestyle (ERL) compared to control placentas. ${ }^{*}$ indicates that the difference between groups was statistically significant $(p<0.05)$.

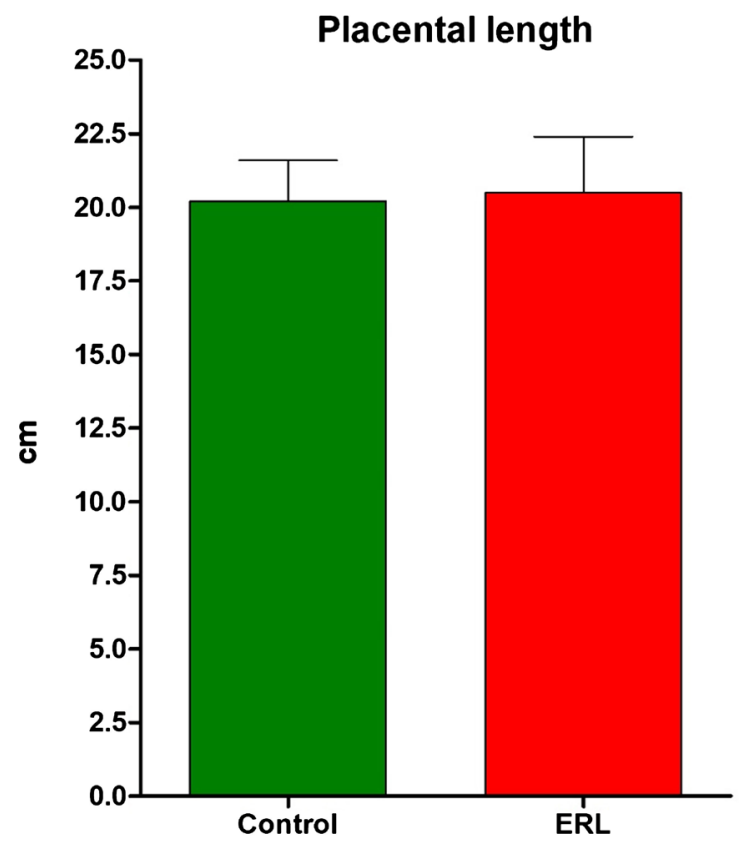

Figure 2. Placental length was comparable between women who exposed to Ramadan lifestyle (ERL) and control. 


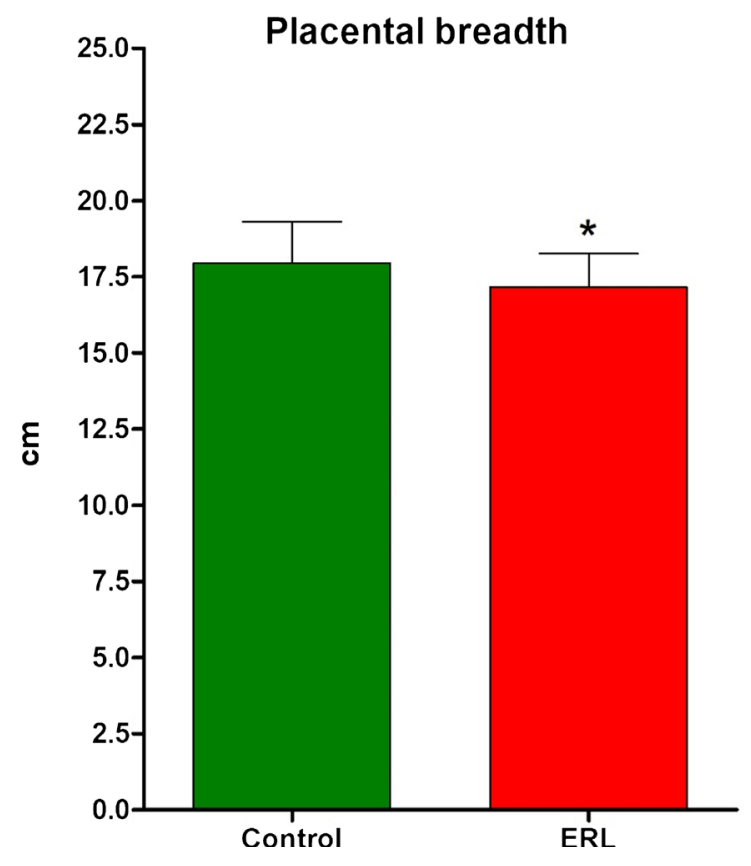

Figure 3. Placental breadth was reduced in women who were exposed to Ramadan lifestyle (ERL) in comparison to control placentas. ${ }^{\star}$ indicates that the difference between groups was statistically significant $(p<0.05)$.

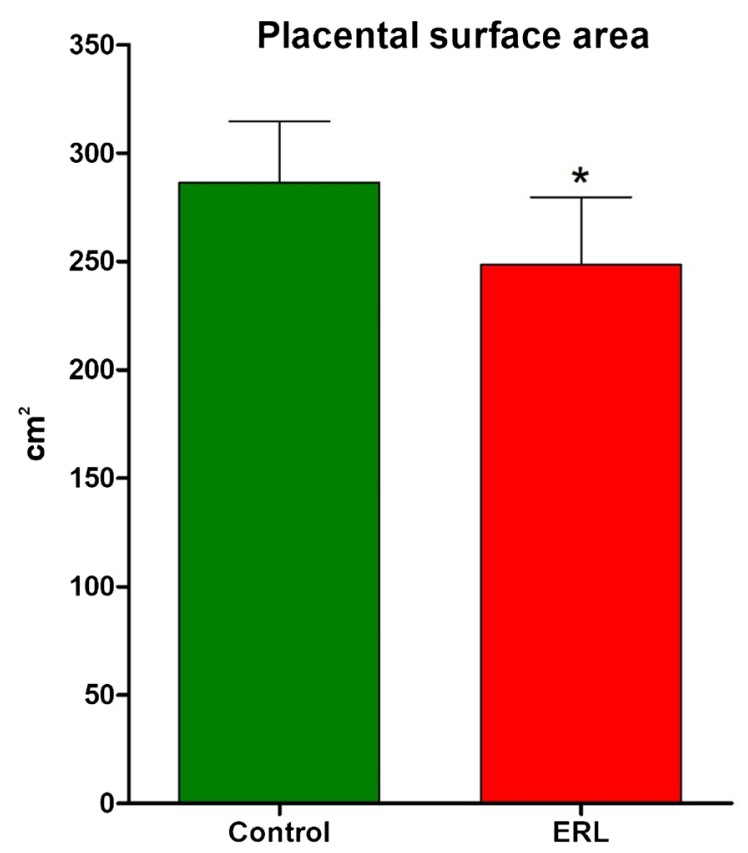

Figure 4. Placental surface area in women exposure to Ramadan lifestyle (ERL) was significantly smaller than control placentas. ${ }^{*}$ indicates that the difference between groups was statistically significant $(p<0.05)$.

became smaller with a very condensed chromatin. In addition, they aggregated densely in some spots forming dark-blue syncytial knots. Placentas from women who were exposed to Ramadan lifestyle, Figure 5(B), seemed to have fewer and less-differentiated syncytial knots. The syncytiotrophoblast cells were markedly thicker and their nuclei tended to be more pleomorphic in some placentas exposed 


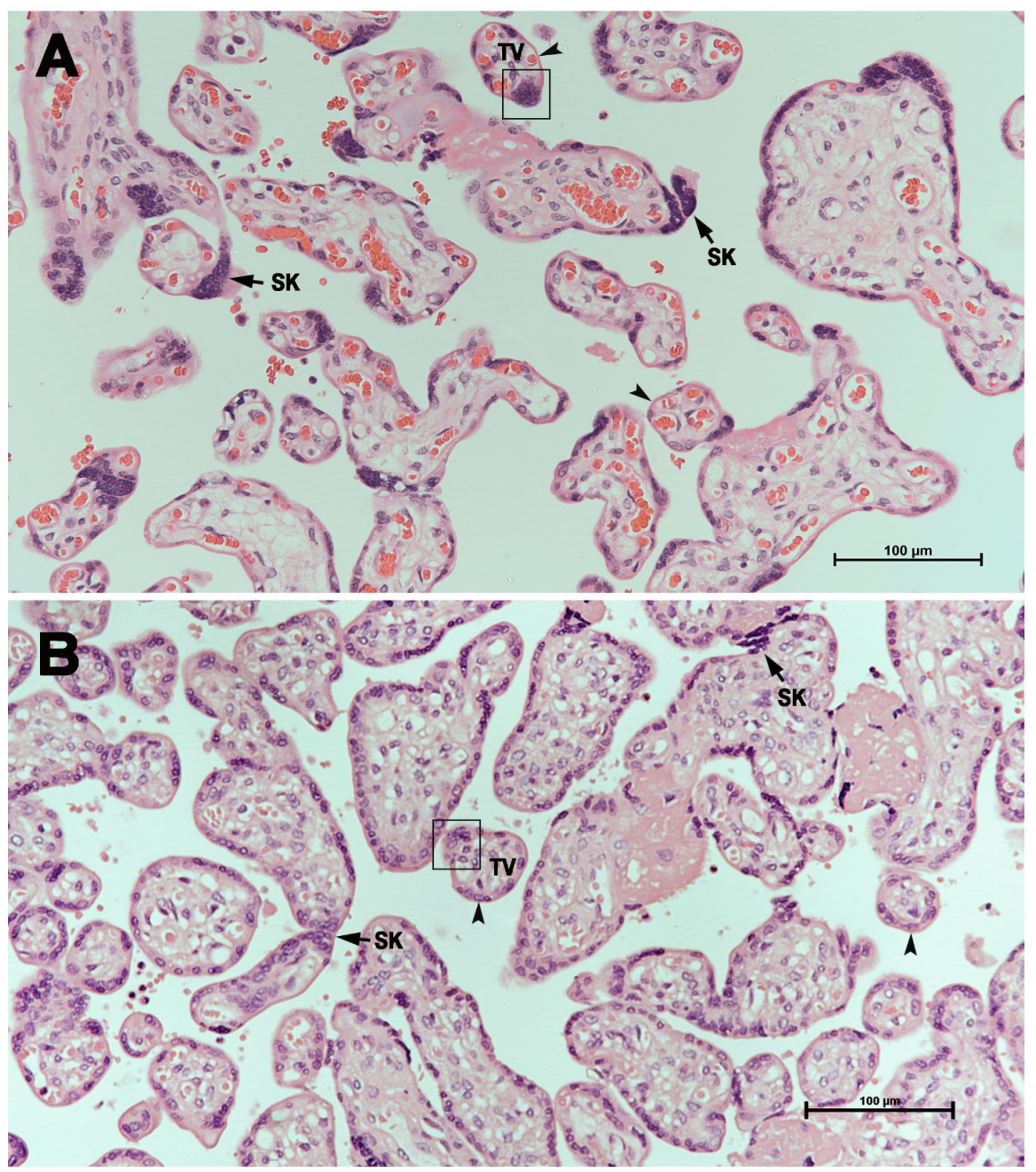

Figure 5. Light microscopic micrographs taken at $20 \times$ magnification. (A) represents not-exposed to Ramadan lifestyle group (control), and (B) represents placentas exposed to Ramadan lifestyle. The syncytial knots (SK) were fewer in placentas exposed to Ramadan lifestyle compared to control placentas. Squares show a terminal villi (TV) with less nuclei aggregation and less-developed syncytial knots in contrast to controls. Cell fusion was absent or uncompleted in the syncytiotrophoblast cells (arrow heads) of placenta exposed to Ramadan lifestyle resulting in a thicker barrier compared to control placentas.

to Ramadan lifestyle in comparison to control placentas. Also, nuclei of syncytiotrophoblast cells in placentas exposed to Ramadan lifestyle were less aggregated and their chromatin was less condensed.

\subsection{DNA Fragmentation}

The TUNEL assay was performed to detect DNA fragmentation as a marker of cellular apoptosis. Figure 6 shows a remarkable DNA fragmentation in the syncytiotrophoblast cells of some placenta from women who were exposed to Ramadan lifestyle in comparison to placentas from women who were not exposed to Ramadan. The placental villous stroma and fetal capillary epithelial cells were negatively stained indicating no DNA fragmentation in all groups. 

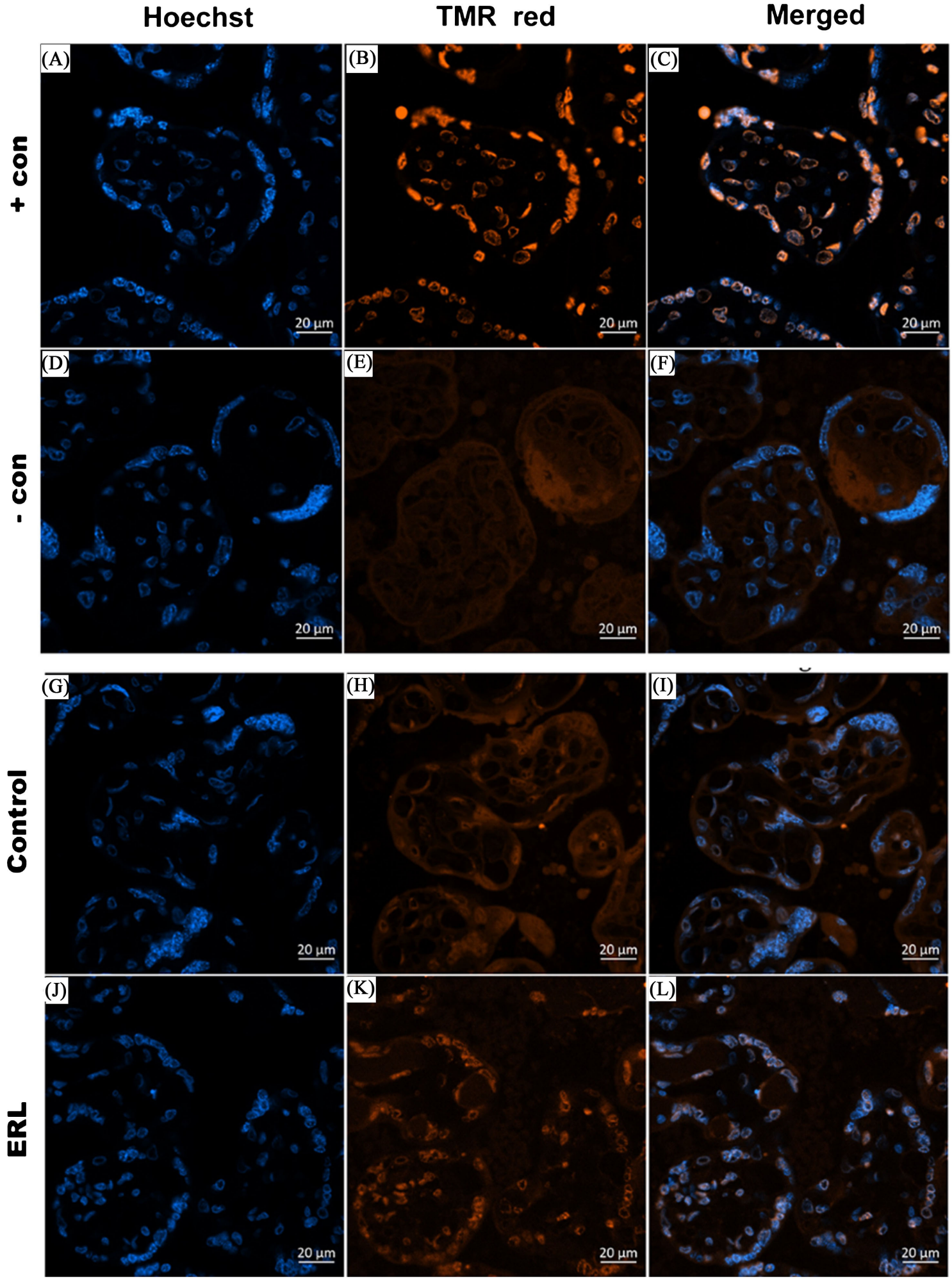

Figure 6. Placental chorionic villi stained with Hoechst and TMR red in the first and second column respectively. The third column represents merged images from the first and second columns. Micrographs (A)-(C) and (D)-(F) show positive (+con, (A)-(C)) and negative (-con, (D)-(F)) controls respectively. Placentas that were not exposed to Ramadan lifestyle (Control, (G)-(I)) did not show TMR red staining indicating no DNA fragmentation. In contrast, the syncytiotrophoblast cells in placentas that were exposed to Ramadan lifestyle (ERL, (J)-(L)) were well stained with TMR red staining indicating that DNA fragmentation and apoptosis were progressing. 


\subsection{Gene Expression of the Placental Glucose Transporter Genes GLUT1 and GLUT3}

Figure 7 and Figure 8 show gene expression of the placental glucose transporters GLUT1 and GLUT3, respectively. The results confirmed that the level of $\beta$-actin gene expression was comparable between the two groups. Gene expression of

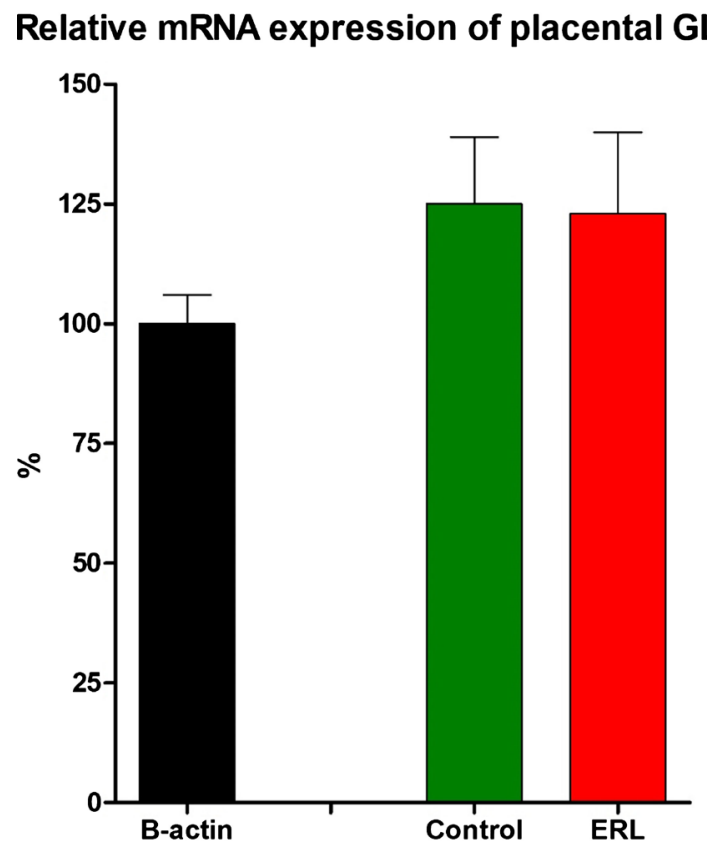

Figure 7. Gene expression of placental GLUT1. There were no significant differences between women who exposed to Ramadan lifestyle (ERL) and control.

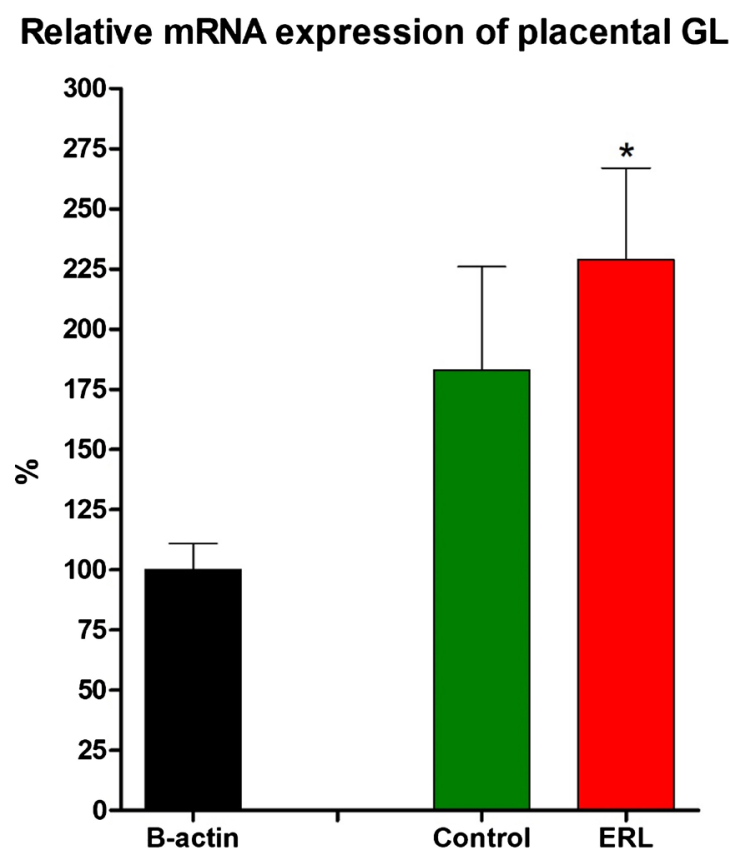

Figure 8. The gene expression of placental GLUT3 was significantly increased in women who were exposed to Ramadan lifestyle (ERL) compared to controls. *indicates that the difference between groups was statistically significant $(p<0.05)$. 
GLUT1 was not affected by exposure to Ramadan lifestyle. In contrast, GLUT3 gene expression was significantly upregulated $(p<0.001)$ in placentas from women who were exposed to Ramadan lifestyle compared to control placenta.

\section{Discussion}

In this study, we have examined the effects of lifestyle during Ramadan on placental morphometrics, histology and glucose transporters expression among Saudi pregnant women. As the placental and fetal growth depend on the mother's nutrient stores and metabolism as well as on her daily food intake [34], changes in maternal lifestyle and physical activity can alter placental development [35].

During Ramadan, pregnant women in Saudi Arabia change their lifestyle dramatically. They consume no food or water during the daytime, which lasted up to 14 hours in the current study. Typically, family members gather before sunset waiting for "Iftar" to break their fasting. Iftar contains a variety of dishes rich in carbohydrates and fats. The next major meal is "Sahoor," which is usually eaten between midnight and dawn. Sahoor meals are rich in proteins and fats but with less sweets compared to the Iftar meal. The school day and business working hours are shortened. Students and employees leave home at 10:00 am until 3:00 pm. Most people stay awake at night and shift their daily activities to night time.

The remarkable change in lifestyle has affected the placenta in the current study in terms of its morphometrics, histology and gene expression. The placental weight in the current study was reduced in women exposed to Ramadan lifestyle. This is consistent with our previous findings in Unizah, a different city in Saudi Arabia [22]. Interestingly, the mean placental weight in the current study was $10 \%-20 \%$ higher than that in Unizah City. In a larger number of placental records collected over a decade in Unizah hospital, it was found that the mean placental weight of Saudi women was markedly rising over time. The authors suggested that the increase in placental weight over the years reflects changes in maternal body composition due to changes in lifestyle as a result of globalization. Small babies generally have small placentas [36]. This reflects that a small placenta has a reduced ability to transfer nutrients, leading to a smaller baby. Experimental studies showed that a suboptimal diet during pregnancy decreases placental weight before changing fetal growth [15]. It seems that the placenta compensates for environmental challenges by changing its structure and/or function. Such changes in placental weight, shape or molecular structure can program the fetus from the earliest stages of development [37] [38].

The placental surface area was reduced in women who were exposed to Ramadan lifestyle. The reduction in placental surface area was mainly driven by a reduction in the breadth but not the length of the placenta. To our knowledge, this the first study to report detailed placental diameters and surface areas for women exposed to Ramadan lifestyle. The surface area measured by calculating the length and breadth does not necessarily reflect the total exchange surface area, but it is reasonable to use it as an indicator because the two are well correlated [34]. The surface area of the placenta reflects the placental growth ability 
and enrichment of the uterine wall. In a study of the Helsinki Birth Cohort, van Abeelen et al. [20] found a significant correlation between small placental surface area and hypertension in later life. Another study found that, in short mothers, babies who were short in relation to their body weight and had either a large or small placental surface area were at greater risk of developing lung cancer [12]. Among the two placental measurements, the breadth was a strong predictor of lung cancer and hypertension [20]. The placental breadth was reduced in women who were exposed to Ramadan lifestyle. This may increase the risk of developing of hypertension, cancer or other adulthood disease as suggested by other studies [12] [13]. The prevalence of hypertension in the Saudi population is over 20\% [39]. The incidence of lung cancer in Saudi Arabia has increased around 4-fold since 1990 [40]. The correlation between incidences during fetal life and later chronic diseases in the Saudi population remain poorly investigated.

The syncytiotrophoblast cells that is in direct contact with maternal blood are thought to be responsible for nutrients exchange. In normal placentas, the syncytiotrophoblast cells fuse and their nuclei become very condensed to form syncytial knots. This leaves a plenty of very thin syncytial cytoplasmic layer that is very efficient for material exchange. In our study, we noticed that the syncytiotrophoblast cells was thicker and the syncytial knots were less-developed in some placentas exposed to Ramadan lifestyle compared to control placentas. In addition, the chromatin of syncytiotrophoblast nuclei was less condensed suggesting active DNA. On the other hand, we noticed some apoptotic indications in the syncytiotrophoblast cells of placentas exposed to Ramadan lifestyle. We speculated that exposure to Ramadan lifestyle may re-programed the syncytiotrophoblast cells delaying cell-cell fusion and thereby delaying the syncytial knots formation and ultimately delaying placental maturation.

The mRNA expression of placental GLUT1 was unaltered by exposure to Ramadan lifestyle. Some cells of the syncytiotrophoblast layer, where GLUT1 is expressed, were positive for TUNEL assay indicating DNA fragmentation and ongoing apoptosis in women exposed to Ramadan lifestyle, however, placentas were still able to maintain normal level of GLUT1 mRNA. Ramadan lifestyle has significantly increased the gene expression of GLUT3. Previous studies reported that GLUT3 is mostly expressed in the second half of gestation to match the dramatic need of the fetus for glucose. Normally, the expression of GLUT3 reflects the current maternal status. In Ramadan, maternal blood glucose gradually decreases during the fasting hours reaching the lowest level before Iftar. Shortly after Iftar, maternal blood glucose is sharply increased due to eating carbohydrate-rich meals [41]. It is reasonable to suggest that placental GLUT3 expression was significantly increased in order to overcome this remarkable fluctuation of glucose availability and to match the increasing demand of the fetus.

\section{Conclusion}

Changes in maternal lifestyle during Ramadan affect the placental structure. The weight of the placenta from women who were exposed to Ramadan lifestyle was 
significantly reduced compared to control placentas. The placental surface area followed the same pattern. The placental length was not affected but the placental breadth was significantly reduced in placentas exposed to Ramadan. It seems that the reduction in placental breadth has driven the reduction in placental surface area and thus placental weight. Exposure to Ramadan lifestyle has delayed the development and maturation of the syncytiotrophoblast layer but in the meantime induced apoptosis. The expected decrease in maternal blood glucose level due to fasting for over 14 hours may have caused an increase in GLUT3 expression in order to match the increasing glucose demand of the fetus. Such findings have been reported to increase the risk of developing adulthood diseases in various human populations worldwide. These compensatory changes in the placenta may have long-term health consequences in Saudis, however, this has not been investigated. Further studies are required to evaluate the possible link between exposure to Ramadan lifestyle in utero and the risk of developing adulthood chronic diseases in the Saudi population.

\section{Acknowledgements}

The authors would like to thank Deanship of Scientific Research (DSR) at king Saud University for funding and supporting this research through the initiative of DSR Graduate Students Research Support (GSR). The authors would like to thank Mr. Waleed S. Alzeyaid for his kind help in tissue processing.

\section{Conflicts of Interest}

The authors declare no conflicts of interest regarding the publication of this paper.

\section{References}

[1] Benirschke, K. and Kaufmann, P. (1995) Pathology of the Human Placenta. 3rd Edition, Springer-Verlag, New York, 871 p. https://doi.org/10.1007/978-1-4757-4196-4

[2] Yampolsky, M., et al. (2008) Modeling the Variability of Shapes of a Human Placenta. Placenta, 29, 790-797. https://doi.org/10.1016/j.placenta.2008.06.005

[3] Chakraborty, D., et al. (2016) HIF-KDM3A-MMP12 Regulatory Circuit Ensures Trophoblast Plasticity and Placental Adaptations to Hypoxia. Proceedings of the National Academy of Sciences of the United States of America, 113, E7212-E7221. https://doi.org/10.1073/pnas.1612626113

[4] Sadler, T.W. and Langman, J. (2006) Langman's Medical Embryology. 10th Edition, Lippincott Williams \& Wilkins, Philadelphia, $371 \mathrm{p}$

[5] Ellery, P.M., et al. (2009) Evidence for Transcriptional Activity in the Syncytiotrophoblast of the Human Placenta. Placenta, 30, 329-334. https://doi.org/10.1016/j.placenta.2009.01.002

[6] Loukeris, K., Sela, R. and Baergen, R.N. (2010) Syncytial Knots as a Reflection of Placental Maturity: Reference Values for 20 to 40 Weeks' Gestational Age. Pediatric and Developmental Pathology, 13, 305-309.

https://doi.org/10.2350/09-08-0692-OA.1 
[7] Harding, J.E. (2001) The Nutritional Basis of the Fetal Origins of Adult Disease. International Journal of Epidemiology, 30, 15-23. https://doi.org/10.1093/ije/30.1.15

[8] Eriksson, J.G., et al. (2000) Early Growth, Adult Income, and Risk of Stroke. Stroke, 31, 869-874. https://doi.org/10.1161/01.STR.31.4.869

[9] Eriksson, M., Raikkonen, K. and Eriksson, J.G. (2014) Early Life Stress and Later Health Outcomes-Findings from the Helsinki Birth Cohort Study. American Journal of Human Biology, 26, 111-116. https://doi.org/10.1002/ajhb.22502

[10] Estampador, A.C. and Franks, P.W. (2018) Precision Medicine in Obesity and Type 2 Diabetes: The Relevance of Early-Life Exposures. Clinical Chemistry, 64, 130-141. https://doi.org/10.1373/clinchem.2017.273540

[11] Friedman, D. and Luyckx, V.A. (2019) Genetic and Developmental Factors in Chronic Kidney Disease Hotspots. Seminars in Nephrology, 39, 244-255. https://doi.org/10.1016/j.semnephrol.2019.02.002

[12] Barker, D.J., et al. (2010) The Prenatal Origins of Lung Cancer. II. The Placenta. American Journal of Human Biology, 22, 512-516. https://doi.org/10.1002/ajhb.21041

[13] Barker, D.J., et al. (2013) The Shape of the Placental Surface at Birth and Colorectal Cancer in Later Life. American Journal of Human Biology, 25, 566-568. https://doi.org/10.1002/ajhb.22409

[14] Barker, D.J. (2007) The Origins of the Developmental Origins Theory. Journal of Internal Medicine, 261, 412-417. https://doi.org/10.1111/j.1365-2796.2007.01809.x

[15] Jansson, T., Thordstein, M. and Kjellmer, I. (1986) Placental Blood Flow and Fetal Weight Following Uterine Artery Ligation. Temporal Aspects of Intrauterine Growth Retardation in the Guinea Pig. Biology of the Neonate, 49, 172-180. https://doi.org/10.1159/000242528

[16] Hafner, E., et al. (2003) Placental Growth from the First to the Second Trimester of Pregnancy in SGA-Foetuses and Pre-Eclamptic Pregnancies Compared to Normal Foetuses. Placenta, 24, 336-342. https://doi.org/10.1053/plac.2002.0918

[17] Longtine, M.S. and Nelson, D.M. (2011) Placental Dysfunction and Fetal Programming: The Importance of Placental Size, Shape, Histopathology, and Molecular Composition. Seminars in Reproductive Medicine, 29, 187-196. https://doi.org/10.1055/s-0031-1275515

[18] Valero De Bernabe, J., et al. (2004) Risk Factors for Low Birth Weight: A Review. The European Journal of Obstetrics \& Gynecology and Reproductive Biology, 116, 3-15. https://doi.org/10.1016/j.ejogrb.2004.03.007

[19] Niu, Z., et al. (2015) Placenta Mediates the Association between Maternal Second-Hand Smoke Exposure during Pregnancy and Small for Gestational Age. Placenta, 36, 876-880. https://doi.org/10.1016/j.placenta.2015.05.005

[20] van Abeelen, A.F.M., et al. (2011) The Sex-Specific Effects of Famine on the Association between Placental Size and Later Hypertension. Placenta, 32, 694-698. https://doi.org/10.1016/j.placenta.2011.06.012

[21] Alwasel, S.H., et al. (2012) The Breadth of the Placental Surface But Not the Length Is Associated with Body Size at Birth. Placenta, 33, 619-622. https://doi.org/10.1016/j.placenta.2012.04.015

[22] Alwasel, S.H., et al. (2010) Changes in Placental Size during Ramadan. Placenta, 31, 607-610. https://doi.org/10.1016/j.placenta.2010.04.010

[23] Hay, W.W. (2006) Placental-Fetal Glucose Exchange and Fetal Glucose Metabolism. Transactions of the American Clinical and Climatological Association, 117, 321-339. 
[24] Novakovic, B., et al. (2013) Glucose as a Fetal Nutrient: Dynamic Regulation of Several Glucose Transporter Genes by DNA Methylation in the Human Placenta across Gestation. The Journal of Nutritional Biochemistry, 24, 282-288. https://doi.org/10.1016/j.jnutbio.2012.06.006

[25] Lin, Y., et al. (2012) Effect of Maternal Dietary Energy Types on Placenta Nutrient Transporter Gene Expressions and Intrauterine Fetal Growth in Rats. Nutrition, 28, 1037-1043. https://doi.org/10.1016/j.nut.2012.01.002

[26] Mazurek, D., Lozna, K. and Bronkowska, M. (2020) The Concentration of Selected Elements in the Placenta According to Selected Sociodemographic Factors and Their Effect on Birth Mass and Birth Length of Newborns. Journal of Trace Elements in Medicine and Biology, 58, Article ID: 126425. https://doi.org/10.1016/j.jtemb.2019.126425

[27] Horne, B.D., et al. (2008) Usefulness of Routine Periodic Fasting to Lower Risk of Coronary Artery Disease in Patients Undergoing Coronary Angiography. American Journal of Cardiology, 102, 814-819. https://doi.org/10.1016/j.amjcard.2008.05.021

[28] Anson, R.M., et al. (2003) Intermittent Fasting Dissociates Beneficial Effects of Dietary Restriction on Glucose Metabolism and Neuronal Resistance to Injury from Calorie Intake. Proceedings of the National Academy of Sciences of the United States of America, 100, 6216-6220. https://doi.org/10.1073/pnas.1035720100

[29] Mansi, K.M.S. (2007) Study the Effects of Ramadan Fasting on the Serum Glucose and Lipid Profile among Healthy Jordanian Students. American Journal of Applied Sciences, 4, 565-569. https://doi.org/10.3844/ajassp.2007.565.569

[30] Sakar, M.N., et al. (2015) Ramadan Fasting and Pregnancy: Implications for Fetal Development in Summer Season. Journal of Perinatal Medicine, 43, 319-323. https://doi.org/10.1515/jpm-2013-0289

[31] Glazier, J.D., et al. (2018) The Effect of Ramadan Fasting during Pregnancy on Perinatal Outcomes: A Systematic Review and Meta-Analysis. BMC Pregnancy Childbirth, 18, 421. https://doi.org/10.1186/s12884-018-2048-y

[32] Gul, Z., et al. (2018) Perinatal Outcome among Fasting and Non Fasting Mothers during the Month of Ramadan. Pakistan Journal of Medical Sciences, 34, 989-993. https://doi.org/10.12669/pjms.344.15654

[33] Janzen, C., et al. (2013) Placental Glucose Transporter 3 (GLUT3) Is Up-Regulated in Human Pregnancies Complicated by Late-Onset Intrauterine Growth Restriction. Placenta, 34, 1072-1078. https://doi.org/10.1016/j.placenta.2013.08.010

[34] Eriksson, J.G., et al. (2010) Boys Live Dangerously in the Womb. American Journal of Human Biology, 22, 330-335. https://doi.org/10.1002/ajhb.20995

[35] Jansson, T. and Powell, T.L. (2007) Role of the Placenta in Fetal Programming: Underlying Mechanisms and Potential Interventional Approaches. Clinical Science, 113, 1-13. https://doi.org/10.1042/CS20060339

[36] Case, R.M. and. Evans, D.E (1985) Variations in Human Physiology. Manchester University Press, Manchester, $241 \mathrm{p}$.

[37] Kwong, W.Y., et al. (2000) Maternal Undernutrition during the Preimplantation Period of Rat Development Causes Blastocyst Abnormalities and Programming of Postnatal Hypertension. Development, 127, 4195-4202.

[38] Gluckman, P.D., et al. (2005) Environmental Influences during Development and Their Later Consequences for Health and Disease: Implications for the Interpretation of Empirical Studies. Proceedings. Biological Sciences, 272, 671-677. https://doi.org/10.1098/rspb.2004.3001 
[39] Al-Nozha, M.M., et al. (2007) Hypertension in Saudi Arabia. Saudi Medical Journal, 28, 77-84.

[40] Althubiti, M.A. and Nour Eldein, M.M. (2018) Trends in the Incidence and Mortality of Cancer in Saudi Arabia. Saudi Medical Journal, 39, 1259-1262. https://doi.org/10.15537/smj.2018.12.23348

[41] Baynouna Al Ketbi, L.M., et al. (2014) Diet Restriction in Ramadan and the Effect of Fasting on Glucose Levels in Pregnancy. BMC Research Notes, 7, Article No. 392. https://doi.org/10.1186/1756-0500-7-392 